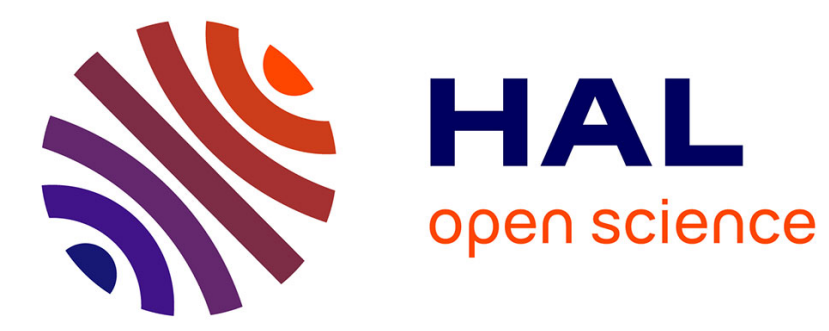

\title{
Noter pour ajuster. Le travail de la scripte sur un plateau de tournage
}

Gwenaële Rot

\section{To cite this version:}

Gwenaële Rot. Noter pour ajuster. Le travail de la scripte sur un plateau de tournage. Sociologie du Travail, 2014, 56 (1), pp.16-39. 10.4000/sdt.4749 . hal-02163359

\section{HAL Id: hal-02163359}

\section{https://hal-sciencespo.archives-ouvertes.fr/hal-02163359}

Submitted on 24 Jun 2019

HAL is a multi-disciplinary open access archive for the deposit and dissemination of scientific research documents, whether they are published or not. The documents may come from teaching and research institutions in France or abroad, or from public or private research centers.
L'archive ouverte pluridisciplinaire HAL, est destinée au dépôt et à la diffusion de documents scientifiques de niveau recherche, publiés ou non, émanant des établissements d'enseignement et de recherche français ou étrangers, des laboratoires publics ou privés.

\section{(이) $\$$}

Distributed under a Creative Commons Attribution - NonCommercial - NoDerivatives| 4.0 


\title{
Noter pour ajuster. Le travail de la scripte sur un plateau de tournage
}

\author{
Noting to adjust. Script supervisor work on a film set
}

\author{
Gwenaële Rot
}

Institutions et dynamiques historiques de l'économie et de la société (IDHES), UMR

CNRS 8533, université de Paris Ouest Nanterre, 200, avenue de la République, 92001

Nanterre cedex, France Disponible sur Internet le 18 f'evrier 2014

\section{Résumé}

Le tournage d'un film est caractérisé par la discontinuité des étapes de fabrication et la survenue de nombreux aléas, ainsi que par un foisonnement d'initiatives non programmées et l'émergence de controverses liées au tâtonnement créatif. C'est à la scripte qu'incombe la tâche de veiller à la «continuité du film », dont dépend la qualité du montage et la vraisemblance de ce qui sera projeté sur l'écran. Une intense activité scripturale lui incombe alors pour garder en mémoire les étapes de fabrication. Ces écrits sont la trace «utile » d'une activité complexe qui prend tout son sens dans le dense mais discret travail relationnel prolongeant l'activité scripturale. Nous montrerons en quoi, dans une organisation fragile comme celle du cinéma, la coopération au travail peut être pensée comme une activité métrologique d'ajustement participant à l'œuvre de création.

Mots clés : Organisation par projet ; Ajustement ; Cinéma ; Écrits ; Erreur ; Travail concret

\begin{abstract}
The act of shooting a film is characterised by discontinuity in the production stages and numerous unpre-dictable events, as well as a host of unscheduled initiatives and the emergence of disputes arising from creative experiment. It is the task of the script supervisor to maintain the "film continuity", which governs the quality of the editing and the plausibility of the final screen product. This demands intense scripting activity to maintain a record of the production stages. These writings are the "effective" trace of a complex activity that achieves its full meaning in the dense but discreet relational work arising out of the scripting process. We will show how, in a fragile organization such as cinema, cooperation in work can be perceived as a metrological activity of adjustment that contributes to the creative process.
\end{abstract}

Keywords: Project-based organization; Adjustment; Cinema; Writings; Error; Practical Work

Adresse e-mail : gwenaele.rot@orange.fr 
«Le cinéma donc, en tant que technique, institution, reflet d'un univers humain, est un fait de civilisation total. Il est une sorte de microcosme, à travers lequel, on peut retrouver — certes déformée, stylisée, ordonnée — l'image d'une civilisation, celle même dont il est le produit. Par là, il relève de la sociologie» (Friedmann et Morin, 1952, p. 95).

En 1952, dans un article de la Revue internationale de filmologie, Georges Friedmann et Edgar Morin établissent les jalons d'un ambitieux programme de recherche de sociologie du cinéma. Reprenant l'argument d'Andrew Buchanan (1951) selon lequel la parcellisation toucherait, fait nouveau, le travail intellectuel et artistique, ils font l'hypothèse que la production cinématographique n'échapperait pas à l'organisation du travail parcellisé dont G. Friedmann (1950) dénonçait déjà les effets délétères ${ }^{1}$. Ils évoquent cependant les limites d'une analyse qui rabattrait l'industrie du cinéma sur le modèle de l'industrie de série, telle l'automobile. Parce que la fabrication d'un film n'exige pas une immobilisation importante de capital, des formes d'organisation artisanales se développeraient tandis que les risques économiques inhérents à cette activité stimuleraient les partis pris créatifs audacieux susceptibles de séduire le public. Toutefois, à l'époque, les analyses de réception ont dominé les recherches en filmologie tandis que les sociologues du travail et des organisations privilégiaient l'étude des grandes bureaucraties industrielles ou administratives.

Il faut attendre les années 1980 pour voir apparaître en France les prémices d'une sociologie du travail cinématographique avec les travaux de Yann Darré (1986) consacrés au cinéma d'auteur. L'intérêt pour l'organisation productive du cinéma est plus affirmé du coté des recherches anglo-saxonnes en théorie des organisations (Mintzberg et McHugh, 1985; Storper, 1989; Baker et Falkner, 1991; De Fillipi et Arthur, 1998; Uzzi et Spiro, 2005; Bechky, 2006; Ebbers et Wijnberg, 2009): en tant qu'activité de création artistique qui s'inscrit dans une organisation par projet (Benghozi, 1989) ${ }^{2}$, le cinéma apparaît comme un secteur emblématique pour penser les transformations des organisations contemporaines (Menger, 2009).

Les études sociologiques de la filière cinématographique attentives au travail concret restent toutefois limitées ${ }^{3}$ pour comprendre les formes et les enjeux de la coopération dans des organisations caractérisées par une intrication étroite des phases de conception et de production, la fragmentation dans le temps et l'espace des étapes de fabrication, l'importance des incertitudes productives.

Au sein de cette organisation, c'est à la scripte qu'incombe la tâche de veiller à la «continuité du film » dont dépend la qualité du montage et la vraisemblance de ce qui sera projeté sur l'écran. Pour ce faire, une intense activité scripturale lui incombe. Dans la perspective d'une sociologie du travail attentive aux modalités concrètes de l'accomplissement des activités professionnelles (Dodier, 1995; Bidet et al., 2006), l'étude du travail de la scripte à travers et à partir de ses écrits professionnels permettra d'identifier dans quelle mesure l'activité d'ajustement qu'elle accomplit est au cœur du travail. Alors qu'en théorie des organisations la notion d' «ajustement mutuel » popularisée par Henry Mintzberg ([1979] 1982) est souvent ramenée à de la simple communication informelle qui viendrait supplanter ou compléter l'organisation formelle pour répondre à des besoins d'intégration, nous proposons d'aborder l'ajustement sur un plateau de tournage comme pratique productive à part entière, dont la mise en œuvre ne va pas de soi.

\footnotetext{
${ }^{1}$ Ils déplorent ainsi le sort des comédiens « contraints de jouer par fragments incohérents un film dont ils n'ont souvent aucune idée d'ensemble» (Friedmann et Morin, 1952, p. 97).

2 Qualifiée aussi par ces auteurs d'organisation «flexible», « de prototype », « latente », « temporaire».

${ }^{3}$ Il importe de signaler l'ouvrage de l'ethnologue Emmanuel Grimaud (2004) qui a remarquablement suivi, en tant qu'observateur participant, l'ensemble des étapes de fabrication d'un film en Inde à Bollywood. Pour un aperçu d'autres facettes du travail cinématographique, en lien avec le programme de recherche que nous avons développé sur le travail cinématographique, voir Seroussi, 2006; Rot, 2007 ; Rot et de Verdalle, 2013.
} 
Le dictionnaire de l'Académie française propose dans sa première édition (1694) des définitions utiles à l'analyse : «Ajustement : action par laquelle on ajuste, on rend juste. L'ajustement d'un poids, d'une mesure, d'une machine. Il signifie aussi accommodement. Il faut travailler à l'ajustement de ces deux personnes. Il signifie encore Parure. Il n'a pas de beaux habits, mais l'ajustement y supplée. L'ajustement fait tout ». Dans sa sixième édition (1832), le dictionnaire propose une acception supplémentaire: «Il se dit encore de la disposition, de l'arrangement d'une chose, de manière que ses diverses parties forment un tout régulier, agréable ». Dès lors, comment s'articulent les différents registres d'action qui caractérisent le travail de la scripte, pensé ici comme activité d'ajustement? En quoi les écrits de travail peuvent-ils être un utile analyseur pour comprendre les spécificités de cette activité ?

Après avoir décrit la configuration de l'espace professionnel et organisationnel dans lequel évoluent les scriptes (section 1), nous montrerons que ce travail doit être saisi dans une double dimension : d'une part à travers la mobilisation d'écrits, analysée ici comme un travail organisationnel qui oriente la vigilance et prépare l'action sur le tournage (section 2), et d'autre part à travers un travail relationnel reposant sur l'observation et l'évaluation professionnelle des manières de faire d'une équipe de film sur un plateau (section 3).

\section{Méthodologie}

Cette enquête s'appuie sur trois types de sources: des entretiens réalisés auprès d'une vingtaine de scriptes françaises débutantes, expérimentées ou très expérimentées, ayant travaillé sur des longs métrages de cinéma de genres différents, à gros ou petits budgets, et d'autres professionnels du cinéma (réalisateurs, maquilleurs, habilleurs, chefs opérateurs-cadreurs, chefs machinistes, etc.) ${ }^{4}$; des observations de tournage (sur une douzaine de films, une trentaine de jours de tournage); la consultation de 25 scénarios et d'autres écrits de travail communiqués par les scriptes rencontrées ou archivés à la bibliothèque du film de la cinémathèque française.

En effet, après le dernier clap, les scriptes conservent souvent leur scénario annoté auquel sont parfois rajoutés des signes plus personnels: autographes, photos des membres de l'équipe décorent parfois la couverture et signalent qu'une aventure est terminée. Les scriptes sont souvent attachées à cet album, un bel objet de surcroît. Mais à la suite d'une réflexion collective ${ }^{5}$, certaines ont décidé de déposer «leurs» scénarios à la Cinémathèque française. Ce geste contribue à affirmer le caractère créatif de leur contribution à l'activité cinématographique.

Les entretiens des scriptes, d'une durée de deux à trois heures, ont été réalisés soit à leur domicile, soit chez moi, soit à la cinémathèque. La plupart du temps ils ont été menés scénario en main. Le scénario est en effet un véritable dispositif activateur de mémoire, qui s'offre aussi comme témoin de l'accomplissement d'une activité complexe.

\footnotetext{
${ }^{4}$ Une trentaine de personnes ont été interviewées dans le cadre d'une enquête encore en cours sur le tournage des films. Les interviews ne portaient pas spécialement sur le travail des scriptes, mais ce sujet pouvait être abordé lorsqu'était évoquée la question de la coopération avec les autres corps de métier.

5 Au sein de leur association professionnelle, «Les scriptes associés » (LSA), fondée en 2005 et qui comprend quatrevingts membres en 2013 .
} 


\section{Le cadre productif et l'enjeu des raccords}

Un scénario et des rapports tenus à bout de bras ou posés sur les genoux, un appareil photo à portée de main, un chronomètre autour du cou, l'écouteur d'un casque son sur une oreille, assise devant l'écran de retour vidéo (le combo) ou se tenant debout aux aguets, la scripte regarde attentivement les comédiens, prend des notes et s'adresse discrètement au réalisateur (ou à la réalisatrice) ${ }^{6}$. Entre deux prises, on la voit se plonger dans ses papiers et à nouveau écrire mais aussi prendre des photos et se déplacer pour échanger à voix basse quelques mots avec un comédien, l'ingénieur du son, la maquilleuse, le cadreur ou encore l'accessoiriste. L'observateur extérieur d'un tournage saisit difficilement ce qui s'écrit et ce qui se dit dans ces moments de forte concentration et d'échanges furtifs. Pour mieux comprendre ce qui se joue dans cette «vie de plateau », il importe de situer la scripte par rapport au cadre général de la division du travail cinématographique (1.1) et aux conventions professionnelles qui guident son action (1.2).

\subsection{La scripte dans la division du travail cinématographique}

La fabrication d'un film est le fruit d'un travail collectif faisant intervenir une multitude de métiers tout au long d'une longue chaine de fabrication allant de la phase d'écriture à la postproduction en passant par le tournage et le montage. Sur le tournage, le réalisateur est entouré de plusieurs chefs de poste.

Les statuts et les rôles de chacun sont établis suivant une hiérarchie professionnelle et salariale très codifiée ${ }^{7}$. Au sommet, le directeur de la photographie (ou chef opérateur) est responsable de la réalisation de l'éclairage des scènes; le cadreur (qui parfois est aussi chef opérateur) est responsable du cadrage de l'image mais aussi de «l'harmonie des mouvements», en lien avec le chef machiniste, auquel incombent les déplacements de la caméra. Également «collaborateur du réalisateur», le chef opérateur de son (ou ingénieur du son) assure la captation des sons sur le plateau. Membre de l'équipe décoration, l'accessoiriste est responsable de la préparation et de la disposition des accessoires de jeu, et du positionnement «raccord» du mobilier choisi par l'ensemblier et ses collaborateurs, les régisseurs d'extérieur. Enfin, maquilleurs, coiffeurs et habilleurs doivent veiller, dans leurs champs de spécialité respectifs et sous la responsabilité de leur hiérarchie (chef maquilleur, chef costumier, chef coiffeur), à l'apprêtement des comédiens.

Cette recension rapide des attributions des intervenants avec lesquels la scripte est le plus souvent en contact fait ressortir des responsabilités spécifiques, des hiérarchies professionnelles, des axes de coopération attendus. La division du travail cinématographique consacre aussi le réalisa-

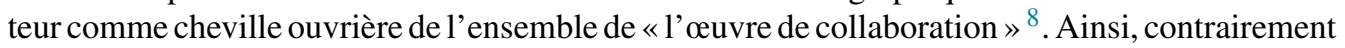
à ce qui est parfois suggéré, la hiérarchie n'est pas incompatible avec l'organisation par projet.

\footnotetext{
${ }^{6}$ Par commodité mais aussi parce que les hommes sont largement majoritaires dans les fonctions de réalisation nous parlerons, dans la suite du texte, du «réalisateur ». À l'inverse et pour la même raison, nous parlons des scriptes au féminin même si, comme nous le verrons plus loin, la profession s'efforce de supprimer cette référence au genre.

7 Pour plus de détails nous renvoyons à la convention collective nationale de la production cinématographique du 19 janvier 2012.

${ }^{8}$ Seule une infime partie des intervenants à l'œuvre collective peut revendiquer le statut d'auteur (Darré, 1986). En droit français, une œuvre audiovisuelle est une œuvre de collaboration. Sont présumés coauteurs d'une telle œuvre: l'auteur du scénario, l'auteur de l'adaptation, l'auteur des compositions musicales, le réalisateur.
} 
Quelle place occupe la scripte dans cette division du travail ? Certaines pratiques d'écriture inscrites dans un cadre professionnel sont considérées comme «secondaires » ou « subalternes », comme ces «tâches d'écriture laissées aux secrétaires, aux assistants, aux petits employés » (Lahire, 1997, p. 147). "Secrétaire de plateau» est d'ailleurs un terme qui était utilisé dans le passé pour désigner les scriptes ${ }^{9}$. La convention collective nationale des techniciens de la production cinématographique du 30 avril 1950 définit son rôle ainsi : «La script-girl: auxiliaire du réalisateur et du directeur de production. Elle veille à la continuité du film et établit, pour tout ce qui concerne le travail exécuté sur le plateau, les rapports journaliers artistiques et administratifs ».

Si cette représentation marque encore négativement l'identité professionnelle des scriptes, la négociation de la nouvelle convention collective des techniciens du cinéma a permis de réévaluer leur rôle:

«Collaborateur technique et artistique du réalisateur. Il fait le lien avec le directeur de production et le monteur, notamment via les rapports artistiques et administratifs. Pendant la préparation, est chargé de pré-minuter le scénario et d'établir une continuité chronologique. Responsable de la continuité, il veille à sa bonne mise en oeuvre pendant le tournage » (Convention collective nationale de la production cinématographique du 19 janvier 2012, Titre II, Chapitre I, article 2).

Entre ces deux définitions, des inflexions sont identifiables : la référence au genre disparaît ${ }^{10}$, et même si «le»scripte ne rentre pas dans la catégorie des «cadres collaborateurs de création » comme les directeurs de la photographie, les créateurs de costumes, les chefs décorateurs ou les chefs opérateurs de son, le rattachement au réalisateur est mis en avant et le mot «artistique » fait son apparition à deux reprises. Sont également rappelés les liens avec l'activité de montage et l'existence d'une phase de préparation. Ces évolutions témoignent de la pression qu'a exercée l'association professionnelle des scriptes pour faire reconnaître la part créative de leur activité $^{11}$.

Il reste que la scripte, bien que «cadre», occupe une place subalterne par rapport aux autres chefs de poste dans la hiérarchie professionnelle et salariale. Son nom n'apparaît pas sur l'affiche et ne figure pas en tête de liste des génériques. Apportant une contribution au film qui ne peut pas être identifiée à l'écran (sauf lorsque sont repérées des erreurs de raccord) ${ }^{12}$, elle semble faire partie de ces «travailleurs de l'écrit» dont l'activité est caractérisée par une certaine invisibilité (Shapin, 1989; Star et Strauss, 1999; Denis et Pontille, 2012). Pourtant, en dehors des tâches d'enregistrement d'informations — comparables à celles d'un greffier — qu'implique le

\footnotetext{
${ }^{9}$ Dans ses mémoires, le producteur et réalisateur Henri Diamant-Berger (1895-1972) indique que c'est suite aux importants problèmes de raccord rencontrés lors du montage de son premier film, tourné en 1915, qu'il décida de solliciter les services d'une secrétaire (Diamant-Berger, 1977, p. 29).

10 Retour du refoulé ? Il est écrit que l'habilleur doit collaborer avec «la » scripte. Ce lapsus présent dans la convention collective de 2012 renvoie au fait qu'il s'agit toujours d'une profession très féminisée. Aux États-Unis, si le terme scriptgirl ou continuity girl était, semble-t-il, d'usage courant dans les années 1930 et 1940, il lui a été préféré celui de $s c r i p t$ supervisor, plus approprié aussi par rapport à celui de script clerc également utilisé (Weldon, 1946, p. 331-333). Sur le caractère «genré » des postes sur un plateau voir Camille Gaudy (2008).

11 Mais cela témoigne aussi de l'importance du travail de préparation. Par ailleurs, un nouveau poste a été reconnu, celui d'assistant scripte.

12 «La scripte, c'est un peu comme le gardien de but au foot: on ne s'aperçoit de son travail que quand elle rate [...]», résumait un réalisateur interviewé.
} 
renseignement de différents rapports ${ }^{13}$, c'est dans une fonction d'agencement scriptural complexe et d'interface relationnelle avec les autres membres de l'équipe d'un tournage que la scripte gère certains risques productifs inhérents au caractère décousu et imprévisible de la fabrication, et contribue ainsi au travail créatif. En effet, contrairement à l'hypothèse avancée par Yann Darré (1986) selon laquelle, depuis la Nouvelle vague, les différents membres de l'équipe technique d'un tournage ne seraient que de simples exécutants de la volonté du réalisateur, le rôle de la scripte est moins passif qu'il n'y paraît.

\subsection{Continuité et raccords}

On ne filme pas les séquences ${ }^{14}$ en suivant l'ordre dans lequel elles seront montrées à l'écran. Pour des raisons d'économie, de disponibilité des lieux et des comédiens, sont tournées la même journée des séquences se déroulant sur un même lieu mais relevant de moments différents du récit. Inversement, des séquences situées dans différents lieux mais censées se dérouler le même jour peuvent être filmées à plusieurs semaines d'intervalle. Par ailleurs, même si le tournage est précédé d'un important travail de préparation destiné à planifier et organiser les différentes étapes de fabrication, le poids des aléas techniques et environnementaux, mais aussi les essais créatifs, conduisent à refaire les plans d'une même séquence soit immédiatement à la suite de ceux qui viennent d'être tournés, soit plusieurs jours voire plusieurs semaines après. La responsabilité de la continuité consiste alors à superviser les raccords afin d'éviter le «choc dans l'œil du spectateur » (Witta, 1949, p. 149).

«Raccorder, c'est assurer la similitude ou la liaison entre des éléments de décor ou de costume, des gestes, des expressions, des scènes tournées à des moments différents. Un comédien lève le bras droit. Dans le contrechamp qui suit immédiatement, il est préférable que ce ne soit pas son bras gauche qui soit levé », expliquait le cinéaste Claude Chabrol (1999 [1976], p. 206). Cette définition didactique mérite d'être précisée en raison de la diversité des types de «faux raccords ».

Les faux raccords les plus faciles à définir sont ceux liés à certains gestes, à l'apparence corporelle des comédiens, au décor, aux objets et accessoires de jeu, ainsi qu'à l'environnement sonore. D'autres portent sur les regards et la direction des déplacements. À leur sujet, il existe une grammaire cinématographique à peu près stabilisée décrite dans les ouvrages de théorie du cinéma (Burch, 1986; Mackendrick, 2010), enseignée dans les écoles de cinéma, apprise sur le terrain lors de stages, et détaillée dans les manuels du métier de scripte ${ }^{15}$. Le théoricien du cinéma Noël Burch les explicite en ces termes :

«Raccord de regard : [...] lorsque le plan A et le plan B montrent séparément deux personnages qui sont censés se regarder, il faut que le personnage "A" regarde vers le bord droit du cadre et le personnage "B" vers le bord gauche, ou inversement. Car, si, dans deux plans successifs, les personnages regardent tous les deux vers le même bord du cadre,

\footnotetext{
${ }^{13}$ Le rapport image est destiné à la phase de post-production ; le rapport production, adressé à la Production, retrace le déroulement temporel du tournage (heures de début et de fin de tournage, pauses, heures supplémentaires et tous les incidents qui ont pu enrayer son bon déroulement); le rapport montage est destiné au monteur. Tous ces documents ne se valent pas pour les scriptes. C'est surtout le rapport montage, où sont mentionnées des appréciations sur la qualité des plans et identifiés ceux qui sont «bons à monter », qui trouve d'abord grâce à leurs yeux : il est la courroie de transmission des décisions de tournage vers le montage. Elles le mobilisent également pour le suivi de la continuité.

${ }^{14}$ Une séquence comprend la plupart du temps plusieurs plans (une même scène est tournée sous des angles différents).

15 Voir notamment Baudrot et Salvini, 1995. Pour une analyse cognitive de la perception de la continuité par les spectateurs voir Berliner et Cohen, 2011.
} 
l'impression inévitablement produite sera qu'ils ne se regardent pas, et le spectateur sentira que son appréhension de l'espace du décor lui échappe tout à coup. [...]

Raccord de direction : [...] un personnage ou véhicule qui sort du cadre par la gauche pour entrer dans un nouveau cadre censé montrer un espace voisin ou consécutif devra obligatoirement entrer par la droite ; sinon, il donnera l'impression d'avoir changé de direction » (Burch, 1986, p. 21).

D'autres raccords concernent la direction d'acteur et la mise en scène : raccords de voix, de ton, ou encore de rythme comme lorsque sont tournés plusieurs plans de déplacement dans un itinéraire à différents jours d'intervalle.

Chaque type de raccord relève de corps de métiers spécifiques : ceux de l'équipe décoration, de l'équipe « habillage, maquillage coiffure » (HMC), de l'équipe lumière, ou encore ceux de la mise en scène. Mais c'est à la scripte qu'incombe la supervision d'ensemble. C'est donc dans le cadre d'une division du travail à la fois technique et morale (Hughes, 1996) que s'inscrit cette mission globale de suivi de la continuité : par délégation partielle, elle partage la responsabilité de la gestion des erreurs de raccord sur un plateau de tournage.

La scripte ne peut pas tout identifier, tout voir, tout enregistrer. L'enjeu est de distinguer, entre les détails sans importance, ceux dont on peut considérer qu'ils ne se verront pas à l'écran et ceux qui peuvent avoir une incidence sur la conception d'ensemble de l'image. Certains risquent de produire un effet de «dislocation », à l'instar des détails de peinture analysés par Daniel Arasse (1996), et sont susceptibles de casser une harmonie ou au contraire de produire une rupture qui enrichira de manière inattendue l'œuvre cinématographique. L'appréciation des détails n'est donc pas qu'une affaire technique.

\section{Les écrits de travail au service de la construction de l'attention}

C'est en suivant l'activité des scriptes à partir de l'usage qu'elles font de leur scénario annoté que l'on peut identifier les moyens qu'elles emploient pour regarder, percevoir, scruter mais aussi identifier et apprécier de possibles accrocs du tournage qui pourraient menacer la continuité de la représentation cinématographique. Cette activité s'organise en deux temps puisque le travail sur le plateau est précédé d'une phase de préparation. Annoté au fur et à mesure, le scénario change progressivement de nature en incarnant non plus le projet de film mais le film en train de se faire (2.1). C'est à partir de ce travail d'équipement que s'effectue la surveillance de la continuité du film qui, loin d'être solitaire, est aussi une affaire collective (2.2).

\subsection{Traces de scriptes sur scénario : écritures de préparation, écritures de tournage}

Tout au long de la préparation du film et du tournage, l'objet principal du travail de la scripte est «son» scénario. Malgré la création récente de logiciels informatiques spécifiques pour tablettes, les scriptes continuent à privilégier un usage sous forme papier dont les contours ont peu varié au cours du temps. Écrit ou co-écrit par le réalisateur ou un scénariste, le scénario se présente sous la forme d'une centaine de feuilles imprimées au recto et reliées. L'histoire dialoguée est découpée en séquences numérotées qui se succèdent selon l'ordre du déroulement logique du récit. Ces séquences sont présentées en respectant un certain nombre de conventions graphiques: les noms des personnages sont en majuscule ; une ligne de titre mentionne l'indication des lieux (extérieurs, intérieurs), le moment (jour, nuit) et l'intitulé du décor. Aucune référence n'est faite à la «réalité » (les vrais lieux de tournage, le nom des acteurs). 
Des didascalies donnent parfois des indications — souvent succinctes - sur les déplacements des comédiens, les éléments de décor, ainsi que sur les meubles ou accessoires de jeu utilisés.

Anticipant les nombreuses manipulations dont il fera l'objet durant le tournage, certaines scriptes relient leur scénario avec des spirales en métal et une couverture cartonnée épaisse. Ce document d'organisation accompagne la scripte tout au long de ses déplacements. Il s'agit un document professionnel «pour soi », même s'il doit pouvoir être transmis à une autre scripte en cas de remplacement et peut être, de temps en temps, consulté par d'autres membres de l'équipe.

La phase de préparation se traduit par une «prise en main » du scénario, qui relève aussi d'une appropriation temporelle puisque l'une des premières missions de la scripte consiste à évaluer, à la lecture du document, la durée du film. Dès cette phase, elle est amenée à déceler des anomalies ou des oublis dans la construction du récit ou sa durée, qui peuvent conduire à une révision du scénario. Lors du tournage, le pré-minutage réalisé devient un repère quotidien pour suivre, chronomètre en main, la durée réelle des plans filmés afin de déceler d'éventuels problèmes de rythme.

Au scénario la scripte ajoute (en la collant ou en la glissant à l'intérieur) la feuille de continuité qu'elle a préparée et qui déroule le film séquence après séquence en suivant l'ordre de la chronologie du tournage, conformément au plan de travail élaboré par le premier assistant à la réalisation. Cette «continuité» prend la forme d'un tableau, composé de différentes colonnes dont l'une est réservée aux raccords où sont reportés le détail des éléments de coiffure ou de maquillage (en particulier les blessures), mais aussi les accessoires et les costumes. En effet, le scénario n'indique pas toujours avec précision, pour chaque séquence, l'état physique et vestimentaire du personnage ni toutes les composantes du décor (nombre de véhicules, de figurants, etc.). Le scénario et la feuille de continuité, documents inséparables, constituent l'armature à partir de laquelle la scripte oriente son regard et sollicite ses interlocuteurs.

«Avant de tourner une scène je regarde ce que j'ai mis dans la continuité, je regarde cette case magique qui est celle des raccords. Plus c'est préparé et moins je fais de bêtises. Parce que dans une grosse journée, on peut passer sans transition de la séquence 6 à la 4 donc on est là aussi pour aider les comédiens et leur dire qu'il s'est passé ça avant, qu'il va se passer ça après. Tout cela, c'est préparé à l'avance dans ce fameux tableau. Après, pendant le tournage, je rajoute des choses ; mais il est évident que plus je prépare, plus je suis béton » (Scripte expérimentée).

L'appropriation du scénario en phase de préparation passe aussi par une mise en forme visuelle (personnages soulignés par une couleur, surlignage de certaines actions), véritable cartographie nécessaire au suivi du cours de l'action cinématographique. «Voir d'un coup d'œil» : l'enjeu est de s'affranchir, sur le plateau, de la contrainte temporelle que suppose la lecture du scénario.

Ensuite, lors du tournage, l'attention de la scripte porte sur les décors, les parures, les dialogues, mais aussi sur les déplacements dans l'espace des comédiens et de la caméra.

Des listes de vêtements et d'objets, inscrites en face de la page dialoguée, sont dressées avec des codes distinctifs en séparant, par exemple, vêtements et accessoires par le rajout d'un astérisque. Les objets sont décrits par leur couleur, leur matière, leur forme, mais aussi leur position, bref leurs traits distinctifs qui permettront de réaliser des contrôles de correspondance le plus simplement possible. 


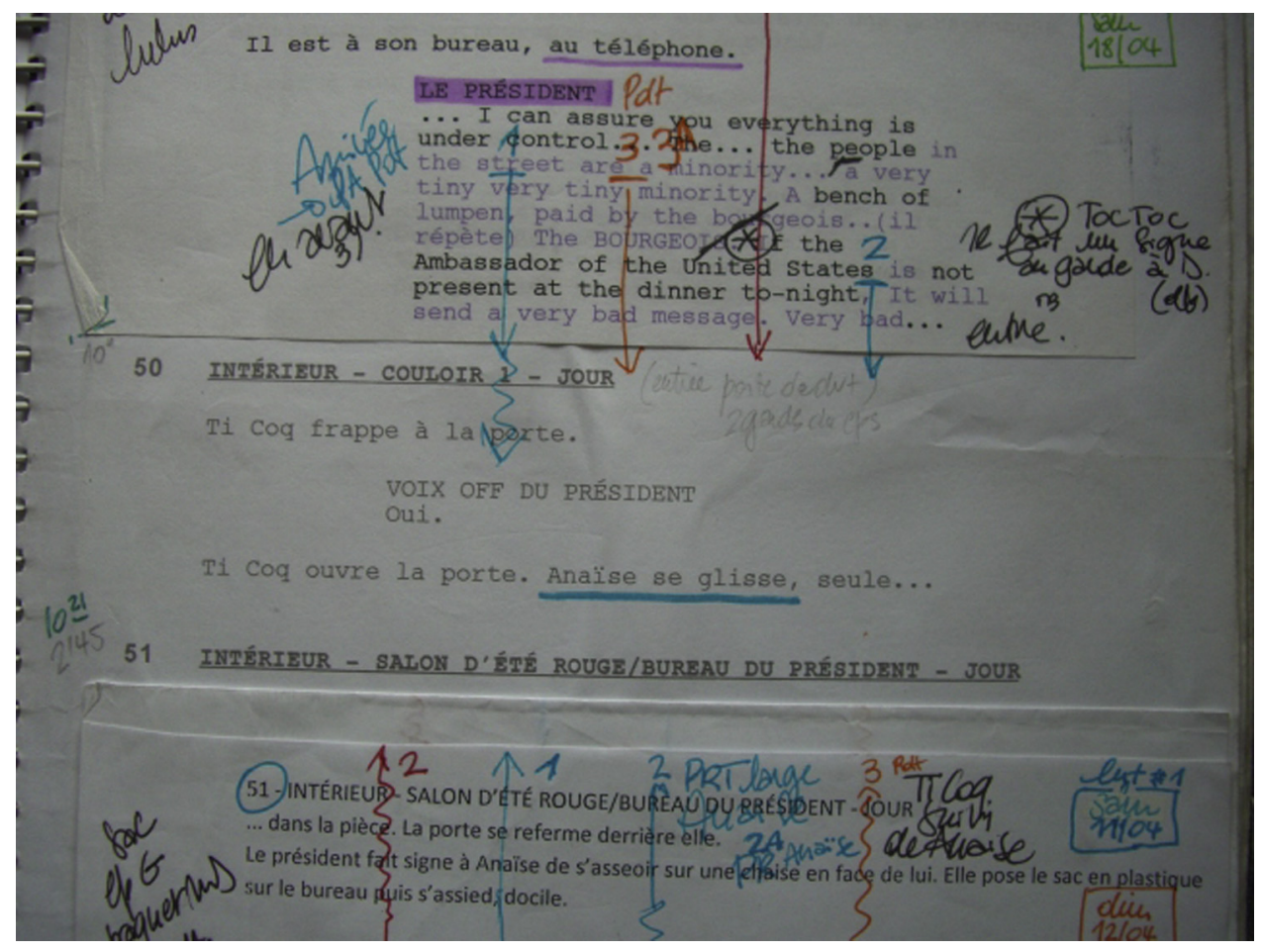

Fig. 1. Les tâtonnements du processus de fabrication se devinent à travers l'épaisseur du papier liée à la superposition des collages lorsque de multiples modifications ont été apportées sur le scénario. Photo de l'auteur.

Lorsque des changements ont lieu à la dernière minute dans le découpage d'une scène, le choix d'un costume et de son ajustement, ou encore le rajout d'un accessoire, les scriptes doivent alors s'assurer, grâce à la feuille de continuité, que ces modifications ne sont pas incompatibles avec les contraintes de raccord des scènes déjà filmées. Parce qu'un dialogue ne passe pas dans le jeu, parce qu'une proposition originale d'un comédien a été retenue, ou encore parce que le réalisateur (parfois suivant la suggestion de la scripte) a pu décider de raccourcir certains dialogues trop longs qui pèsent sur la dynamique de la scène, ou revoir la chorégraphie des déplacements, les rectifications apportées doivent être enregistrées. Les listes récapitulatives (de scènes tournées, de scènes non tournées, de scènes à tourner, de scènes supprimées, etc.) fournissent autant de points d'appui pour se repérer, recenser le travail accompli, constituer des signaux d'alerte... et se rassurer.

Ainsi, les scénarios portent la trace des différents styles de réalisateurs : ceux qui exigent un respect scrupuleux du texte et qui n'admettent pas ou peu d'avenants, ou ceux pour qui le travail d'ajustement des dialogues et de la mise en scène est l'objet de tâtonnements tout au long des prises (Fig. 1).

La scripte retranscrit aussi schématiquement certains modes opératoires en vue de reconstituer des techniques de prise de vue: angles dessinés sous forme de deux traits partant d'un point pour indiquer l'orientation de la caméra, croix pour positionner la place des meubles, croquis signalant la position des acteurs et flèches indiquant leur trajectoire (Fig. 2). Des petits dessins (soleil, nuages), parfois colorés, rappellent le contexte météorologique du tournage de la scène. 

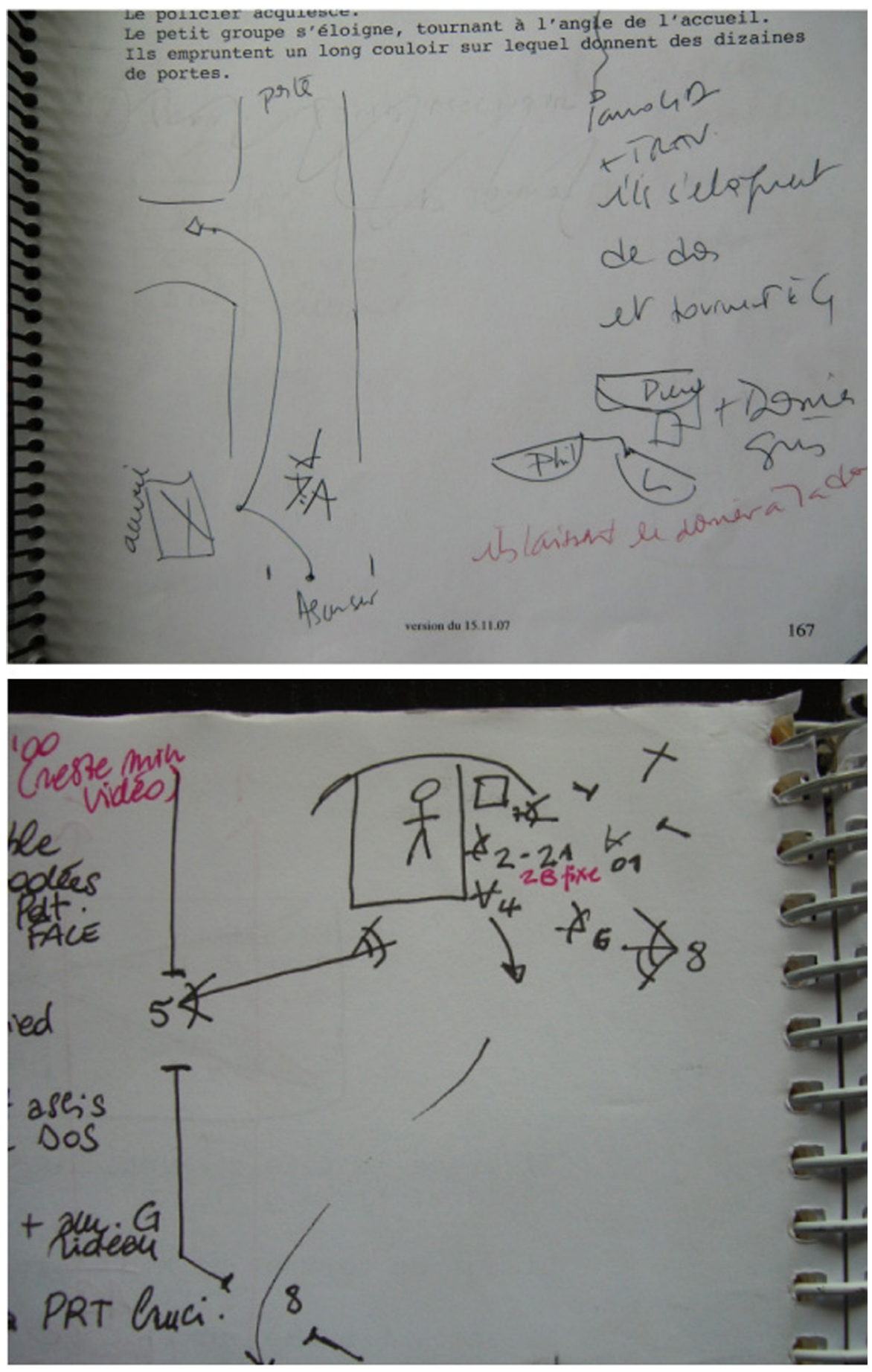

Fig. 2. Représentation des schémas de déplacement. Photos de l'auteur. 
Des traits verticaux ou ondulés, de taille variable, strient les pages écrites du scénario afin de représenter la longueur de chaque plan tourné pour une même séquence. Des écritures abrégées identifient les cadrages et complètent les schémas désignant les mouvements de caméra ou les trajectoires du comédien (de la gauche vers la droite (« GD»), de la droite vers la gauche («DG»)). La scripte doit être attentive à la distribution spatiale des acteurs et des objets qui se trouveront face à la caméra, ainsi qu'à celle des mouvements et des axes.

Par rapport à d'autres activités de retranscription telles que celles des sténographes parlementaires décrits pas Delphine Gardey (2010), le travail de la scripte consiste non seulement à traduire de l'oral vers l'écrit, mais aussi du visuel vers l'écrit et du visuel vers le visuel : les syntagmes que livre la langue naturelle ne permettent pas de décrire de manière concise et précise des combinaisons de mouvements. Le «détour de production d'écriture » est souvent incompatible avec le temps bref dont disposent les scriptes dans le cours de l'action. La notation est sélective, schémas et photographies se substituant parfois aux mots car les rythmes de la transcription et de l'objet de la description ne concordent pas.

Le scénario ne renferme pas toutes les informations utiles à la scripte pour garantir la continuité. Il est prolongé par d' autres instruments de mémorisation tel le combo ${ }^{16}$, et surtout l'appareil photo. En effet, afin de saisir « le détail des détails » (Piette, 1996), des gros plans photographiques sur des mains baguées, des parures, des blessures ou des plaques d'immatriculation s'imposent (Fig. 3).

En général, les photos sont faites après la prise, mais le silence des appareils numériques permet d'en prendre aussi au cours du tournage, notamment en photographiant l'écran du combo, technique qui garde la mémoire du cadrage et la valeur du plan. Les photos ne «parlent » pas toujours par elles-mêmes. «Privées d'un principe de marquage, [elles] sont des signes qui ne prennent pas bien, qui tournent comme du lait », écrivait Barthes (1980, p. 18). Les scriptes opèrent tant bien que mal leur classement, même si celui-ci semble plus difficile à opérer avec l'accumulation d'images provoquée par la photographie numérique ${ }^{17}$.

«La façon d'écrire, le choix d'une graphie contribuent [...] à la signification du message » et à ses effets performatifs, rappelle Béatrice Fraenkel (2006, p. 9). Les scriptes sont toujours attentives à la composition d'ensemble du document: la mise en page, l'usage de couleurs, la manière de coller des photos ne sont pas laissés au hasard. Grâce à cette mosaïque de références, traces éclectiques qui «brutalisent le complexe, le trient et l'ordonnent » (Dagognet, 1973), le scénario se lit comme un espace organisé qui facilite des mises en relation, les repérages dans l'espace et le temps. C'est ainsi qu'il rend possible une «prise» sur le déroulement du tournage. En retour, ces techniques d'enregistrement organisent la perception, comme l'a montré Goodwin (1994) — qui étudiait le travail de notation des archéologues dont le métier est, sur bien des points, comparable à celui des scriptes, puisqu'il s'agit pour eux aussi de garder la trace d'une activité passée afin d'en permettre, ultérieurement, la représentation.

«Reconstituer le Rubik's Cube», «retomber sur ses pattes»: les expressions utilisées par les scriptes pour décrire ce cheminement vers un retour au lisible traduisent bien leurs préoccupations lorsqu'elles écrivent, mais aussi dessinent et photographient. Ces inscriptions polymorphes sont des techniques d'assurance, «au cas où » un comédien ne reproduirait pas le même geste ou ne

\footnotetext{
16 Écran sur lequel il est possible d'observer «au cadre » la scène en train d'être tournée et de « re-» voir cette scène le cas échéant.

17 Sur les polaroïds — qui permettaient une quasi-instantanéité entre la prise de photo et sa manipulation matérielle —, le numéro des plans et des séquences pouvait être inscrit immédiatement sur chaque photo. Avec le numérique, lorsque les photos ne sont pas imprimées, le classement s'opère par exemple en photographiant la page du scénario concernée avant de prendre les photos qui correspondent à celles de la séquence.
} 


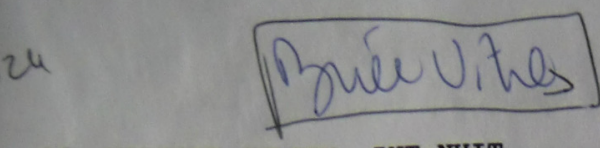

\section{VOITURE JULIEN. INT-NUIT}

La tête basculée sur le repose-tête, il fixe le plafond de la voiture.

À ses côtés se trouve le sac en toile : Celui-ci est vide. Complètement abattu, Julien soupire le visage sombre.

Dehors retentit le bruit sourd d'un autoradio de voiture.

Julien tourne la tête pour voir à travers sa vitre embuée le cabriolet rouge du dealer qui s'éloigne.
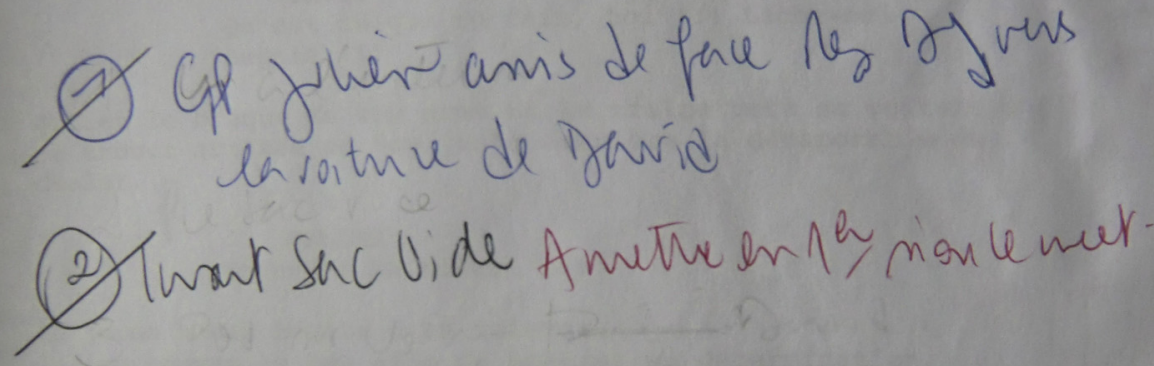

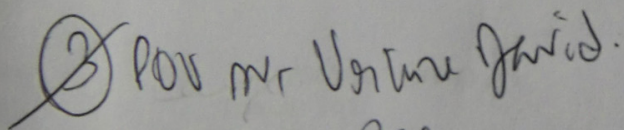

- avir muore

- Sara anove.
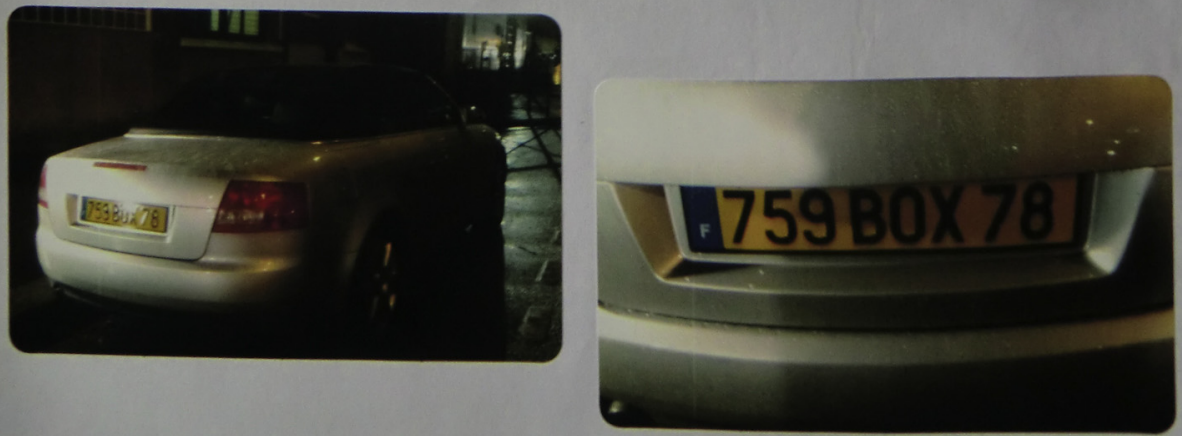

Fig. 3. La «saisie» des détails: des photos d'un véhicule et de sa plaque d'immatriculation complètent les écritures abrégées qui indiquent les types de plans tournés. Photo de l'auteur. 
porterait pas l'accessoire dans la bonne main, «au cas où » le chef opérateur oublierait le type de filtre utilisé, «au cas où » l'accessoiriste aurait un doute sur la place des objets positionnés, « au cas où » il faudrait prévoir une seconde prise «à l'identique ». Les scriptes tissent les mailles d'un filet de sécurité pour une organisation très exposée aux risques d'erreurs et d'oublis. Ces opérations de relève des détails sont donc loin d'être mécaniques. Elles dépendent de conventions professionnelles mais aussi du contenu artistique du film et des modalités concrètes de tournage. Enfin, cette activité est tributaire du microcosme social, chaque fois singulier, qu'est le plateau de tournage.

\subsection{La construction de la vigilance, une affaire de mobilisation collective}

Le tournage mobilise des spécialistes inscrits dans une "chaîne de coopération » (Becker, 1988) bien balisée. Chacun est chargé de concentrer son attention sur un domaine d'intervention particulier : la mise en scène, la lumière, les décors, le son, le maquillage, la coiffure, l'habillage, etc. La scripte occupe une position au cœur de la coordination de ces domaines de spécialité. Sur le terrain, des conventions sont établies pour s'accorder dans le cours de l'action et favoriser un travail collectif de contrôle.

«Je m'adresse souvent à l'habilleuse pour savoir comment elle note. Entre un pantalon noir et un pantalon un peu noir on essaye de se mettre d'accord sur les dénominations. L'habilleuse me dit "tu n'as qu'à marquer que c'est le pull machin" et elle me donne la marque, et moi j'ai la marque car c'est comme ça qu'elle le reconnaît. Après, moi je peux lui dire : “aujourd'hui c'était tel pull”, en lui donnant la marque » (Scripte expérimentée).

Les écrits circulent, se complètent. La plupart des scriptes communiquent leur feuille de continuité à leurs différents interlocuteurs concernés par les «raccords». Certaines vont plus loin, comme cette scripte qui, chaque nouvelle journée de tournage, fait le point avec les principaux chefs de poste de l'équipe, à l'instar d'un commandant de bord se préparant pour le décollage de son avion:

«Au début du tournage du film je distribue à chaque département une copie de ma continuité. Je leur dis que le matin je passerai toujours les voir. Sur la feuille de service ${ }^{18}$ je reporte [à partir de la feuille de continuité] les choses qui concernent l'humeur, des particularités de raccords de costumes, blessures. . . Chaque fois qu' on change de séquence, je revérifie cette feuille de service, que je connais par cœur. Je me fais des doubles check, des triples check. Le matin, en arrivant sur le plateau, je vais voir tous les départements, avec cette feuille dans la main, sur laquelle j' ai noté les choses [...], en disant : "Bon, alors là, on est bien d'accord, cette scène se passe pendant une bagarre donc, est-ce que tu sais exactement où tu vas te faire une blessure ?". On en parle avec le comédien, avec le réalisateur, avec le maquilleur, avec le cascadeur. Donc, anticiper un maximum, continuer à poser des questions, à vérifier, à contrôler $[\ldots]$ » (Scripte très expérimentée).

Tout au long du tournage, l'espace d'observation de la scripte est situé dans un triangle balisé par trois principaux outils: le combo, la caméra, la roulante de l'ingénieur du son. Sa place implicitement «réservée » et partagée avec le réalisateur est, la plupart du temps, derrière le

\footnotetext{
18 La feuille de service, élaborée par le premier assistant à la réalisation, est communiquée la veille pour le lendemain à tous les membres de l'équipe. Elle détaille le planning de la journée.
} 
combo. Le réalisateur lui livre ses premières appréciations sur la qualité du jeu, sur le rythme de l'agencement de la scène... et aussi ses états d'âme ${ }^{19}$. Dans ces moments d'échanges privilégiés, il lui demande son avis — ou de manière plus implicite guette une approbation, scrute ses réactions - pour sélectionner les plans avant qu'elle ne les mentionne sur le rapport destiné au montage. Mais le réalisateur n'est pas son seul interlocuteur.

À l'instar des contrôleurs de RER décrits par Isaac Joseph (1994), l'attention de la scripte oscille entre «attention distribuée et attention focalisée », exercice qui n'a rien d'évident :

«Cette actrice, j' ai besoin de rien lui dire pour les raccords. Elle est tout le temps raccord! C'est-à-dire qu'elle est super-juste dans les rythmes, les gestes, les regards, l'expression, c'est une Rolls. Ce n'était pas comme les autres qui étaient assez mauvais. À un moment, dans une séquence, j'avais tous mes comédiens, dont mes "bons" et mes "pas bons", et le problème c'est que j'ai regardé la bonne : je vois S. [l'actrice "Rolls"] me faire un truc très compliqué et je me dis "hum, c'est compliqué ce que tu fais là, est-ce que tu vas réussir à le refaire ?". Et puis-je la regarde attentivement et elle le refait tout le temps, et pendant ce temps les autres qui avaient des choses plus simples ont fait n'importe quoi ! (Scripte peu expérimentée).

Les scriptes interviewées insistaient sur le fait que leur confort de travail dépendait beaucoup du professionnalisme des autres techniciens. L'ajustement consiste alors aussi à construire la fiabilité du système de fabrication, quitte à orienter, voire à rectifier le travail des autres.

«Le problème, ce sont les actrices aux cheveux longs qui se touchent tout le temps les cheveux. En plus de regarder leurs textes, leurs mouvements, leurs déplacements, il faut aussi regarder comment elles se touchent les cheveux ! En général en répétition tu vois tout de suite quand tu pars en live par rapport à ça. Dans ces cas-là j' appelle le coiffeur et je lui dis: "viens, regarde ce qu'elle fait parce qu'elle touche beaucoup ses cheveux". Cela fait partie de leur boulot, je leur rappelle qu'il faut qu'ils soient dessus parce qu'ils ne le font pas toujours et ils attendent trop la scripte. Je l'appelle pour qu'il vérifie quand ce sont des choses compliquées. Car nous on a tellement de choses à noter sur un même plan que cela devient. . . La queue de cheval qui passe en avant et après qui passe à l'arrière, c'est voyant à l'image, c'est gênant au montage » (Scripte expérimentée).

Savoir à qui l'on a affaire est déterminant pour hiérarchiser ses priorités d'attention, mais aussi pour définir les contours de la coopération avec les autres membres du collectif. Identifier les acteurs qui «ne savent pas tenir leur raccord», voir comment se débrouille un membre de l'équipe HMC débutant, etc., importe pour régler son juste positionnement dans la manière d'organiser ce travail de surveillance-contrôle, sachant par ailleurs qu'une confiance trop assurée est un facteur de risque :

\footnotetext{
19 On peut retrouver dans le «couple» réalisateur-scripte des similitudes avec la situation décrite par Josianne Pinto (1990) au sujet des liens entre «la secrétaire et son patron», notamment quand elle écrit: «[les secrétaires] rendent possible l'activité du patron en s'ajustant de façon presque inconditionnelle à ses qualités les plus indéfinissables, à commencer par son humeur ou ses humeurs sans lesquelles il ne serait pas ce qu'il est. Cet ajustement [...] est fait d'une multitude d'actions et de réactions réciproques ainsi que d'un travail sur soi pour corriger des aspects non appropriés à un contexte déterminé » (p. 36). L'efficacité attendue de qualités considérées comme «naturellement féminines » (la méticulosité, la discrétion), rappelle Pinto, serait la garantie d'un travail «accompli sans discussion, débat ou critique sur le fond » (ibid.). Il en va toutefois différemment de la scripte dont on attend qu'elle signale des dysfonctionnements sous réserve de ne pas brider inutilement le travail créateur.
} 
«Quand on est en confiance, c'est aussi là qu'on lâche le morceau et c'est là que vous, vous êtes en confiance avec l'habilleuse, et la comédienne n'arrive pas avec le bon pull ! En fait, il faut toujours rester vigilant et c'est cela qui est dur par moments. C'est quand on est dans un moment de relâchement que les conneries se passent » (Scripte expérimentée).

La critique faite, lors des entretiens, à l'encontre de ceux et celles qui n'ont pas leur matériel (appareil photo) ou ne tiennent pas de carnet personnel, et du coup font des erreurs ou sollicitent trop la scripte, révèle un autre enjeu : celui de la construction d'une division du travail pour le suivi des raccords. En effet, être entourée d' interlocuteurs en qui elle peut avoir confiance allège la partie la moins noble de l'activité de la scripte (le contrôle des raccords de vêtements, d'accessoires, de coiffures) et la rend davantage disponible pour ce qui relève du registre plus valorisant de la mise en scène.

À cet égard, une forte complicité dans la coopération a été observée avec les ingénieurs du son. Les échanges, toujours menés dans la discrétion — parfois par un simple jeu de regards ${ }^{20}$ —, portent sur l'identification d'un bruit extérieur au moment d'une réplique, sur la conformité au scénario de ce qui est dit par le comédien, mais encore sur la précision et le ton juste de la prononciation, les raccords de rythme des paroles, le volume de la voix. Le croisement des avis compte pour conforter une impression. Cette confrontation des évaluations de la qualité d'une prise facilite le tri et la hiérarchisation de la masse d'informations à traiter. La scripte se trouve aussi moins seule lorsqu'il s'agit de faire part d'un problème au réalisateur ou au comédien.

Tout au long du tournage, le travail de veille se précise. La scripte «devine le problème du voisin », sait « aller à la pèche », « laisser traîner une oreille » (Joseph, 1994, p. 564). La manière d'exercer cette surveillance, et de se connecter au reste du plateau, est guidée par la mémoire des expériences marquantes (erreurs de raccords du passé) que l'on a soi-même «éprouvées » lors de précédents tournages ou dont on a eu connaissance lors d'apprentissages à l'école ${ }^{21}$.

Savoir où « voir » pour superviser la continuité du film, c'est aussi savoir écouter. Les scriptes ont un casque de retour son qui les relie aux comédiens. Certaines préfèrent ne mettre qu'un écouteur afin de «rester connectées avec le reste du plateau», comme nous l'a expliqué l'une d'entre elles ${ }^{22}$. Mais se «connecter au plateau» implique aussi de gérer ce qui se prépare avant la prise, par exemple en demandant à l'ingénieur du son de ne pas couper le micro des acteurs entre deux prises :

«Ces “oreilles", comme je les appelle, elles sont censées donner le retour [son] uniquement pendant la prise, donc toi tu as toujours le scénario et tu suis que les mots soient dits à la virgule près dans certains cas... ou pas à la virgule près dans d'autres... Entre les prises l'ingénieur du son, en principe, coupe les micros. Quand il voit qu'il peut me faire confiance, il peut me laisser le micro ouvert, ou alors je lui demande de me laisser mon retour son, pas pour entendre ce que les comédiens se disent entre eux, parce que franchement j'en n'en ai rien à faire, mais plutôt pour entendre ce que le réalisateur va leur dire. C'est aussi une

\footnotetext{
20 «Le voir humain et son travail permanent d'anticipation tactique sont à l'oeuvre dans pratiquement tous les champs du social », rappelait à juste titre Véronique Nahoum-Grappe (1998, p.70). Dans l'espace ouvert du plateau de tournage, où les prises de parole sont très contrôlées, le rôle des regards est déterminant dans le travail d'ajustement continu qu'implique l'activité collective. Ils fonctionnent bien comme « un commentaire permanent de l'interaction elle-même» (ibid.).

${ }^{21}$ Formation à la Fémis (École nationale supérieure des métiers de l'image et du son) ou dans des écoles de cinéma privées.

${ }^{22}$ Cet écouteur peut être différent de celui de l'ingénieur du son, moins perfectionné, plus léger, moins isolant pour lui permettre ce braconnage d'information.
} 
de mes façons de ne pas m'insérer dans ce moment privilégié qu'il peut y avoir entre un comédien et un réalisateur, mais d'être au courant quand même pour savoir où l'on va. Cela peut être très utile sur une intention de jeu [...]. Une fois j'ai entendu le réalisateur dire au comédien: "à ce moment-là tu ne sais pas telle chose". Or il faisait une erreur... donc ce que j'ai fait? Je me suis approchée discrètement d'eux et là on a remis les pendules à l'heure » (Scripte expérimentée).

Par ses observations attentives, la scripte identifie erreurs et manquements qui sont autant de signes d'imperfections professionnelles; elle reçoit, de manière volontaire ou involontaire, des confidences sensibles, en premier lieu celles du réalisateur appréciant le jeu d'un comédien. C'est la raison pour laquelle, en raison de la position d'interface qu'elle occupe, la scripte a accès, d'une certaine façon, à des savoirs coupables. Le traitement de ces savoirs est au cœur de son métier.

\section{La scripte dans l'action : signaler et répondre}

Le scénario, tel qu'il est travaillé par la scripte dans un cadre collectif, devient un véritable investissement de forme (Thévenot, 2006) qui participe à la lisibilité de son environnement professionnel et qui rend possibles des « recadrages » lorsque des ruptures de continuité sont identifiées. Il est précieux également lorsqu'il s'agit de répondre aux sollicitations de ses interlocuteurs. Ces actions sont tributaires d'un système social avec lequel il faut composer. Solliciter (3.1) ou être interrogée (3.2) sont les deux facettes du travail relationnel de la scripte.

\subsection{Intervenir dans le tournage}

Un réalisateur interviewé résumait en ces termes les raisons de sa connivence avec sa scripte:

«Elle défend le film souvent alors que toutes les autres personnes sur un tournage, non. L'assistant réalisateur il défend le plan de travail; le chef op., lui c'est son cadre ou sa lumière, le son c'est le son. Elle, c'est le film, c'est la bonne boussole » (Réalisateur).

Mais la scripte «défend» le film également par rapport à ses propres règles professionnelles. Aussi, identifier le détail problématique ne suffit pas : il importe de faire reconnaitre que celui-ci «compte» au regard d'échelles de valeur qui ne sont pas partagées de manière homogène par tous. Des discordances portent sur l'identification de ce qui compte. Elles donnent lieu à des négociations - pacifiques lorsque chacun s'efforce de prendre en compte la contrainte et le point de vue de l'autre pour composer avec lui, ou plus conflictuelles lorsque les représentations de ce qu'est un raccord acceptable ne parviennent pas à converger. Ainsi, avec les chefs opérateurscadreurs, des négociations sont susceptibles de porter sur les axes de regard, de dynamique de cadre, de raccord de plan à plan.

«C'est souvent sur des trucs de cadre: t'as un plan large, et puis on fait un gros plan, allez hop on va remonter tel comédien par rapport à tel autre parce que la différence de taille qui fonctionnait dans le plan précédent, cela serait mieux qu'elle soit atténuée. Donc on va déplacer les objets et les personnes. On passe notre temps à tricher ! C'est là qu'il y a parfois des discussions avec la scripte ("là on peut tricher", "non ça se voit", "non ça ne se voit pas”, etc.). Le chef op. a plus souvent gain de cause car c'est lui qui fait. . . et puis car c'est souvent des mecs qui gueulent plus fort pour avoir raison même s'ils ont tort ! [rires]. C'est moi qui place la caméra où je veux. Donc je ne vais pas me forcer à la mettre où je ne veux pas ! Bon... Mais en général, c'est un accord, on discute, j'essaye de la convaincre : "Viens 
voir dans le cadre, tu verras bien !" Après il y a de la diplomatie : tu te dis "ce coup-là c'est moins important pour moi, je lui donne raison comme cela la fois d'après j'aurai gain de cause" » (Chef opérateur-cadreur expérimenté).

Ce témoignage suggère que la discussion - marquée aussi par les rapports de genre - passe parfois par une confrontation des points de vue, au sens propre du terme. Ici «le cadre de la caméra », convoqué comme force argumentative et combiné avec l'autorité technique et symbolique du chef opérateur, aurait permis à ce dernier d'obtenir gain de cause. Ce récit par un autre chef opérateur de ses démêlés avec une scripte évoque d'autres tensions possibles et une issue différente :

«Le premier jour de tournage elle dit: "ah non mais ça c'est trop large, on ne pourra pas le raccorder". Elle trouvait que ça allait faire une saute avec un plan plus serré. Je lui dis: “bah oui mais justement c'est bien”. On était dans un paysage luxuriant, magnifique, c'était un truc un peu en appel d'air et tout de suite après, il y avait des plans serrés sur les acteurs. Ça faisait un effet. On a alors fait un plan un peu moins large, mais du coup on ne saisit pas du tout l'ampleur du paysage... Quand la scripte disait ça, le réalisateur n'exprimait pas d'idée sur la question. Mais comme la scripte use un petit peu de... comment dire, en fait, elle pense beaucoup au montage. C'est elle qui dit : "non mais là, juste après c'est la séquence untel, juste avant c'est ça, il faut penser au montage, ça va faire vraiment très bizarre". Le réalisateur a été dans son sens car il ne voulait pas du "très bizarre". Elle avait fait deux films avec lui, moi c'était mon premier» (chef opérateur en début de carrière).

En étant capable d'énoncer avec fermeté, scénario en main, les caractéristiques précises des plans filmés et encore à filmer, la scripte a des armes pour convaincre et rassurer un réalisateur hésitant, surtout si celui qui aurait pu avancer un autre point de vue cède, faute d'assurance. Dans ces moments de délibération et de négociation, qui sont aussi l'expression de jeux de pouvoir (Friedberg, 1993), se joue la définition des marges de l'intervention possible par rapport à l'activité créatrice. Dès lors, un commentaire sur la mise en scène, qui peut aider certains réalisateurs, nourrir leur travail, peut aussi être vécu comme une forme d'ingérence :

«Cette scripte-là, je n'avais pas confiance en son jugement artistique sur le jeu des acteurs. La première semaine [de tournage] elle disait: "c'est bizarre" ou "c'est pas bizarre, ça ?". Et je répondais: "si mais c'est fait exprès". Et au début notre relation était un petit peu tendue parce que ça me déstabilisait. C'était sur un geste, une expression... une absence d'information, donc un manque de didactisme aussi peut-être de l'histoire. C'était son rôle d'être aussi un garde-fou, mais quand vous avez une volonté artistique et qu'on vous dit "mais c'est pas un peu bizarre, ça ?", c'est gênant. Elle a dû sentir que ces questions-là ne m'aidaient pas car elle a fini par les poser moins » (Jeune réalisateur, à propos du tournage son premier long métrage de fiction).

On comprend mieux pourquoi, pour la scripte, dire et solliciter expose à la remise en cause de la part de ses interlocuteurs. C'est le cas notamment lorsque la scripte s'adresse au réalisateur ou aux comédiens. Les scriptes interviewées considèrent que c'est la relation avec ces derniers qui est sans doute la plus délicate à gérer. Le récit cocasse d'une scripte à propos de deux occasions de grippage souligne le caractère variable du rapport de force :

«Pour ces détails [raccord de vêtement], en général le réalisateur [un réalisateur chevronné, charismatique qui lui accordait sa confiance et avec qui elle avait travaillé sur plusieurs 
films] me laissait faire complètement, c'est moi qui gérais avec les comédiens [...]. Dans cette scène, V. [un comédien connu] est censé avoir traversé la rivière puis être monté sur la barque, il doit donc avoir un jean mouillé. Mais il n'a pas envie de rentrer dans un jean mouillé ! Il me dit : "mais c'est pas possible ! Ça fait des heures qu'on est là [sur la barque], le jean a séché". Moi : "Écoute, non. Dans la continuité ça ne fait pas des heures, elle est revenue, le temps qu'elle revienne vous avez été drogués, vous êtes au milieu du lac et vous avez le jean mouillé, je n'y peux rien, c'est l'histoire !'. C'est un acteur très intelligent, il a des milliers d'arguments et il essaie de te noyer sous les informations pour que finalement tu dises “ok, fais ce que tu veux !". Et donc j’ai terminé en lui disant: “je n’ai pas envie de t'écouter, ça ne m'intéresse pas. Alors soit tu mets le jean mouillé et tu es raccord, soit tu ne le mets pas et tu n'es pas raccord. Maintenant t'es un grand garçon, c'est toi qui es sur l'image". Alors là, il m'a regardé avec des yeux comme ça. [Elle le mime, les yeux exorbités] "Qu'est-ce que c'est que cette fille qui me parle comme ça ?". Et le réalisateur arrive et il demande : "y a quoi, y a un problème ?". Réponse de V. : "elle me parle mal !"; moi : "je ne parle pas mal, il ne veut pas mettre le jean mouillé, or il doit avoir le jean mouillé dans la scène, voilà !". Et le réalisateur l'a regardé, il lui a dit : "si F. dit que tu dois avoir le jean mouillé, tu mets le jean mouillé, t'as pas le choix". C'était terminé, il a été mettre son jean mouillé [...]. Je peux donner un autre exemple avec le même réalisateur où je n'ai pas été suivie. C. [une actrice] arrive avec un super turban sur la tête. Je vois arriver ce truc de no where, je me dis: "Mais c'est quoi ?" Parce que le rôle ce n'est pas ça : dans le rôle elle est crade, elle a de la poussière partout et elle va mourir, là, dans deux minutes douze ! [rires] C'est pas possible ! Donc je vais la voir : "c'est quoi... ?" Elle : "oui on a décidé ça avec le coiffeur. On a trouvé que pour la scène c'était bien, il n’y a pas de raccord". Le grand truc de tous les acteurs qui veulent faire une chose qui n'est pas permise c'est: "il n'y a pas de raccord !". Je réponds : "bien sûr il n'y a pas de raccord direct, t'es pas sortie de la chambre et tu n'es pas rentrée dans la chambre avec le machin. Il n'y a pas de raccord, mais il y a quand même une cohérence du personnage et on peut quand même se poser la question...". Alors elle me regarde et elle se tourne vers le réalisateur qui l'aime bien: "le foulard là, qu'est-ce t'en penses ?". Lui, à l'autre bout du plateau, il se retourne et lui répond: "Ouais ! Mmm !". Elle me regarde : "Ben voilà, le metteur en scène trouve ça bien”. Dans ce cas c'est fini, tu peux plus. . . Terminé. Fin de l'histoire. Parce que sinon tu rentres en conflit ouvert avec l'acteur et tu mets du coup le metteur en scène à contribution, ça n'a pas de sens. Mon boulot c'est de te dire que ça doit être comme ça car c'est comme ça que ce sera le mieux - enfin [elle se reprend], que cela sera le mieux, dans mon orthodoxie à moi, de mon métier; maintenant si tu m'expliques que toi tu as envie de faire autrement et que le metteur en scène est d'accord. . . c'est son affaire ... » (Scripte très expérimentée).

Etre capable de dire les choses importantes et se taire sur des choses qui ne le sont pas, telle est en substance la ligne de conduite professionnelle qu'évoquent les scriptes lorsqu'elles parlent de leur métier. Leur expérience, la confiance que leur accordent le réalisateur mais aussi certains comédiens et techniciens, leur connaissance accumulée relative aux types de réactions possibles, pèsent sur la sélection qui s'opère dans ce que l'on décide de signaler, et aussi dans la manière de le faire. Mais la frontière est mince entre le signalement d'un problème, perçu comme un soutien, et la révélation d'un manquement professionnel, stigmatisant son auteur et dont la mise au jour risque d'être contre-productive — par exemple en fragilisant un acteur dans son jeu. De même, une suggestion artistique peut être reconnue comme un apport positif à la création ou vécue comme une ingérence malvenue. 
Or, le plateau de tournage est un espace étroit où chacun agit à découvert et où les occasions de perdre la face sont plus fréquentes que dans un espace cloisonné dans lequel le travail se déroule sans interférences permanentes entre travailleurs. La communication y est donc une affaire très délicate : agir avec efficacité consiste à trouver le bon moment pour intervenir ainsi que les mots pour le dire. Dans ce contexte, le tact et la discrétion sont une règle d'or pour faire passer les messages.

«On ne parle pas aux gens de loin. Si par exemple vous décelez un truc de costume qui n'est pas juste, on ne dit pas: "dis donc, c'est pas le bon pull !". On va voir la costumière en disant: "t'es sûre que c'est ce pull-là, moi je...". Et puis bon, ben, des fois vous avez tort, hein. Mais on ne fait jamais perdre la face aux gens » (Scripte expérimentée).

Parce que le plateau de tournage est une formation sociale fonctionnant, d'une certaine manière, comme la société de cour décrite par Norbert Elias, l'art d'observer les détails des objets, des parures, mais aussi l'harmonie d'ensemble du plan tourné, se double donc d'un art d'observer les comportements des hommes ${ }^{23}$.

Ainsi l'accès « direct» au comédien peut-il se révéler difficile, notamment lorsque le réalisateur n'est «pas prêteur», formule utilisée par une scripte interviewée. Il n'est pas rare qu'une comédienne accorde plus de crédit à une remarque d'un chef opérateur, haut placé dans la hiérarchie symbolique des titres et des postes, qu'à celle d'une scripte. Remonter un problème lorsqu'on estime qu'il est d'importance suppose alors de suivre des voies détournées pour ne pas froisser les susceptibilités. Dans cette organisation mi-hiérarchique, mi-collégiale, les contrôles diagonaux sont donc fréquents. S'appuyer sur le relais d'autres interlocuteurs lorsqu'ils ont «l'oreille » de la personne que l'on souhaite atteindre, qu'il s'agisse du réalisateur, d'une star ou du chef opérateur, est pratique courante.

Juger quand l'on peut intervenir et auprès de qui (pour signaler un problème ou faire une suggestion) fait donc partie des compétences des scriptes sur le plateau, univers très segmenté quant à la définition des attributions de chacun et en même temps très ouvert en raison de la singularité des situations de travail. Mais l'évaluation du risque qu'il y a à intervenir sur le déroulement du tournage est à rapporter à l'évaluation d'un autre risque : celui de se voir reprocher, lors du visionnage des rushs ou au montage, des erreurs de raccord. Trouver des solutions alternatives lorsque le respect de certains raccords est trop difficile à obtenir est aussi une piste à envisager. Dans ce cas, les stratégies de compensation proposées affleurent parfois des enjeux de mise en scènes, comme le suggère le témoignage de cette scripte :

«Vous devez être capable de dire : "si on ne respecte pas ce raccord, ce n'est pas montable". Donc si on ne peut pas monter, tout le travail est perdu. Si cela bloque au niveau du comédien qui n'est pas raccord, à un moment il faut. . . il faut intervenir, proposer des choses : "peutêtre qu'on peut faire un contrechamp, peut-être qu'on peut. . . avec une autre focale, ce qui permettra de ne pas voir les mains qui nous posent des problèmes"... C'est demander au metteur en scène de faire son boulot, de trouver une autre solution, mais c'est aussi peut-être lui suggérer de filmer le plan avec un autre angle. Si vous ne dites pas de bêtises trois fois de suite, il a une certaine tendance à vous écouter » (Scripte très expérimentée).

\footnotetext{
23 «Dans l'univers de la cour on regarde l'individu toujours avec ses implications sociales, dans ses rapports avec les autres. [...] Amener l'autre, surtout s'il est d'un rang plus élevé, d'une main légère et sans qu'il s'en rende compte, à penser selon ses propres désirs est le premier commandement du commerce avec les hommes de la cour », écrivait Norbert Elias (1985 [1969], p. 99 et p. 105).
} 
Ce qui importe n'est pas d'avoir «gain de cause» par rapport à la grammaire des règles de raccord, parce que les partis pris créatifs d'un réalisateur peuvent aussi consister à ne pas respecter ces conventions, mais d'avoir averti pour éviter ensuite de porter la responsabilité d'une erreur. Dans ce cas, le recours à l'écrit pour mentionner, sur le scénario et sur le rapport destiné au montage, un choix de mise en scène comme un «saut d'axe», dédouane et soulage :

« [Le fait d'écrire] cela protège, et au moins le monteur est prévenu qu'il y a un faux raccord mais que c'est volontaire en décision. Ce n'est pas la scripte qui a m... Tu te libères de ce truc car ce n'est pas un faux raccord à toi, cela a été validé. Comme ça le réalisateur au montage ou le monteur ne se dit pas "tiens, j'ai un faux raccord et personne ne m'a rien dit” (Scripte expérimentée).

\subsection{Répondre aux sollicitations}

La scripte est interpellée par ses interlocuteurs du plateau lorsque surgit un doute : sur la place d'un accessoire, sur la conformité d'un dialogue, sur le temps qu'il faisait lors du tournage d'un plan, sur le cadrage d'un plan... ou la pertinence du jeu d'un comédien. Un ingénieur du son peut solliciter une scripte à propos de l'appréciation sonore d'une scène. D'autres techniciens attendent son soutien, tel ce chef machiniste qui lui demande de chronométrer le mouvement d'une caméra afin de caler celui-ci sur une partie précise du texte ou d'une action, ou encore ce cadreur qui sollicite son attention pour l'aider à veiller aux «bords cadre » afin de s'assurer que le perchman ne perche pas trop bas ou que le projecteur installé par un électricien ne «déborde pas».

La manière d'être sollicitée n'est pas dissociable de la façon dont la scripte a su ajuster ses modes d'intervention et trouver sa place — place dont la construction est difficile, surtout en début de carrière comme l'évoque cette jeune scripte :

«Moi j'ai un petit peu du mal... ce n'est pas que je suis impressionné mais... je crois que j'ai peur de les déranger, de trop intervenir, et c'est vrai qu'il y en a certains qui ne supportent pas qu' on vienne leur parler de raccord. Moi j'ai tendance à y aller ultra-progressivement. . . Alors que c'est vachement agréable quand un comédien vient te poser une question parce qu'il sait qu'on a été attentif à ce qu'il fait. C'est un lien qui est vivifiant, énergisant, mais je trouve que c'est compliqué » (Scripte peu expérimentée).

Les demandes qui sont adressées à la scripte peuvent être vécues comme une marque de confiance mais aussi comme une épreuve, notamment en début de tournage lorsque certains comédiens, par exemple, «testent » sa capacité à identifier un faux raccord.

Dans ces instants, la qualité de l'agencement scriptural réalisé est mise à l'épreuve. L'appropriation du scénario lors de la phase de préparation facilite la réponse dans le feu de l'action. Mais en cas de doute, répondre aux sollicitations nécessite une enquête élaborée à partir des annotations du scénario, de la feuille de continuité et d'informations conservées dans d'autres documents, tel le rapport montage. C'est en mobilisant les images stockées qu'émerge aussi le «tangible», à savoir «ce qui résiste aux variations perceptuelles, instrumentales et argumentatives auxquelles le soumettent des acteurs dotés de représentations et d'intérêts divergents » (Chateauraynaud, 2004, p. 168) : la photo atteste une position, une coiffure, un accessoire, un détail. C'est ainsi qu'on peut « couper court» au doute et clore d'éventuelles controverses. Grâce aux multiples inscriptions, dessins, photos, abrégés, destinés à reconstruire le fil du film et à imaginer le processus de fabrication, le scénario documenté reste toujours un «point d'attache». 
Mais sa pertinence repose autant sur la qualité de ce qui est inscrit que sur sa capacité à faire sens une fois relié à d'autres dispositifs, y compris des documents élaborés par d'autres membres de l'équipe technique. Le témoignage d'une maquilleuse explicite ce processus de confrontation croisée observable sur un plateau :

«Ah la scripte ! Oh là ! Alors ça c'est super important. [...] J'ai mon scénario où je colle toutes les photos raccord à coté des séquences, où en général j'ai des notes de tout ce que j' ai mis comme produits. Je suis un peu “ma scripte à moi”, mais c'est vrai que des fois entre les prises... Par exemple, s'il y a des couleurs de sang, des couleurs de mascara, un truc qui évolue pendant la scène par rapport aux scènes qui ont été choisies, des fois il faut voir ça avec la scripte pour savoir comment ça coulait dans ce plan-là, celui qu'ils vont monter au final. Donc il faut que dans la suite ça soit comme ça. Et puis pour tous les petits doutes, on confronte nos cahiers... En croisant, c'est comme cela qu' on s'y retrouve, et puis je sais aussi qu'il y a les maquilleuses qui ne tiennent pas leurs raccords, qui vont directement voir la scripte aussi » (Maquilleuse expérimentée).

Et c'est en «sauvant des coups » dans l'arène cinématographique que la scripte s'affirme dans la chaîne de production créatrice. Ces rares instants de félicité sont remémorés avec fierté.

«Avant chaque plan je demande toujours l'objectif, la distance, le filtre et la hauteur caméra. Chose qu'on n'a pas toujours l'habitude de demander. [... . Dans [X, une grosse production], ça m'avait bien servi. Les jours où il y avait les fonds verts, le spécialiste des effets spéciaux notait tout. Mais on a tourné des tas d'autres séquences où il n'y avait pas de fond vert et où il n'était pas là. Or, moi j'ai continué à noter la hauteur caméra. Un jour le réalisateur a voulu changer un figurant, un gamin qui rigolait, il en voulait un qui ne rigole pas. Alors il a fait venir les effets spéciaux pour mettre un autre figurant à la place [et qu'il fallait filmer avec la même technique de prise de vue]. Et alors là, c'est à ce moment-là que le gars des effets spéciaux dit "ah oui, bon d'accord..." Comme il n'avait pas ces informations, il se retourne vers moi :

"Alors l'objectif?

- Je l'ai.

- La distance?

- Je l'ai.

—Le filtre ? Bon oui, S. (moi) l'a noté, très bien ! Et vous auriez la hauteur caméra ?

— Oui j'ai la hauteur!"

Ah Ah !... Il n'espérait pas tant !» (Scripte très expérimentée).

Inversement, l'incapacité à répondre à une sollicitation signe l'échec en mettant au jour une défaillance. Survient alors la situation tant redoutée : «être prise en défaut », «être battue », autant d'expressions entendues — dans tous les corps de métiers — qui traduisent la crainte du stigmate. Dans ces moments délicats, les ruses apprises par l'expérience visent à sauver la face :

«Quand tout à coup on demande "Où est-ce que c'était, à quel moment je me lève ?" et que tu n'as pas la réponse, tu as toute l'équipe qui te regarde: "Comment, elle ne sait pas la réponse ?". C'est très frustrant. . Comment je réponds ? Ah, cela dépend des gens [...]. Soit tu donnes ta réponse, soit tu dis que tu ne sais pas et tu vas vérifier car tu as noté des choses dans le cahier et tu as pris des photos. [...] Si jamais en début de tournage, pour me tester, un comédien me dit: “j' ai pris l'objet de la main droite ou de la main gauche ?”, je lui dis [sans hésiter] : “c'est la main droite”. Et puis, après avoir vérifié : "ah ben non c'était la 
prise trois. . . mais sur la prise quatre tu l'as fait de la main gauche". Il ne faut pas montrer ses faiblesses. Bizarrement, tu n'as pas le droit à l'erreur, alors qu'on ne peut pas tout voir et tout noter!» (Scripte expérimentée).

En définitive on peut dire que ce qui caractérise «l'art de faire » de la scripte sur un plateau de tournage est de savoir tenir un double jeu d'effacement et de présence dans le travail des autres. La difficulté — et l'intérêt — du travail est d'arriver à jouer sur des registres dont l'articulation est délicate: tenir une position de «non personne» qui se manifeste dans le cadre d'un travail d'enregistrement, de transcription et de réponse à des sollicitations ponctuelles, et intervenir dans le cadre d'une position de «personne-présente-qui-sollicite ${ }^{24}$ non seulement les autres techniciens, «personnels de renfort » en arrière-scène, mais également les personnes «à la face » (acteurs, réalisateur). Les scriptes qui dosent mal ce subtil cocktail de discrétion, de renoncement et d'initiative prennent le risque d'agacer et de déclencher l'opprobre, voire de compromettre leur avenir professionnel.

\section{Conclusion}

Le plateau de cinéma est un espace organisationnel fragile et sensible. Prise dans différents horizons temporels de référence, la scripte doit réagir dans l'urgence du moment présent mais aussi se projeter dans le passé de ce qui a été fait et dans le futur du tournage. Étudier les écrits au travail à travers ce qu'ils engagent dans et pour l'action permet de mettre au jour le caractère multi-facettes d'une activité où « la dispersion devient un état de chose, une donnée avec laquelle il faut composer» en permanence (Grimaud, 2004, p. 141), au sein d'une organisation éclatée puisqu'il s'agit aussi de travailler pour le montage. Cette activité repose sur un savoir d'orientation dont l'efficacité réside dans la capacité d'identifier rapidement ce qui ne «fonctionne pas », et d'être en mesure de faire la part des choses parmi un foisonnement de données à traiter. À l'instar de ce qu'a montré Hutchins (1994) en étudiant le déroulement de l'activité des pilotes de ligne dans une cabine de pilotage, la surveillance de la continuité apparaît comme une activité multi-équipée, distribuée et éminemment collective. Au cœur du réseau socio-technique qu'est un plateau de tournage, le scénario-cahier, objet nodal, tient tous les autres en prenant part de manière déterminante à la coordination des différents corps de métier. Ces scénarios annotés sont un témoin de l'activité d'ajustement en même temps qu'ils la rendent possible. Ils représentent les traces utiles d'une activité mais ils ne suffisent pas à en rendre compte dans toutes ses dimensions.

Pour comprendre comment la scripte agit pour garantir la continuité du film qui contribuera à produire cet «effet de réel » propre au cinéma ${ }^{25}$, on voit l'importance du travail d'organisation que rendent possible ces dispositifs d'écriture, et aussi celle du travail relationnel engagé sur le plateau. Nous avons montré, à partir de l'exploration de ce qu'il convient d'appeler les usages combinatoires des écrits de travail, que ce travail d'ajustement dans lequel s'engagent les scriptes — mais aussi leurs interlocuteurs — n'est pas dissociable d'une dense activité d'évaluation des hommes et des objets. Cette métrologie à la fois technique et sociale est consubstantielle au travail productif (Vatin, 2013 [2009]). Les opérations d'appréciation «en contexte» du travail d'autrui sont une nécessité pour pouvoir faire son propre travail. En signalant les faux raccords, en infirmant

\footnotetext{
${ }^{24}$ Cette situation est comparable, sur ce point, avec celle qui est décrite par Hervé Glevarec (2001, p. 113-114), s'inspirant de Goffman (1974), dans sa recherche sur des plateaux d'enregistrement radiophoniques.

25 La scripte n'est pas la seule personne ayant cette responsabilité. Le travail du monteur (ou de la monteuse), qui prolonge, en quelque sorte, celui de la scripte, est aussi déterminant et mériterait de faire l'objet d'autres investigations.
} 
ou confirmant un avis, en suggérant le tournage de nouvelles prises, les interventions de la scripte rassurent ou au contraire distillent l'inquiétude, empiètent sur le territoire professionnel de ses interlocuteurs de façon heureuse ou malheureuse. Le travail d'ajustement inhérent à l'activité productive de fabrication d'un film n'est donc jamais neutre pour ceux qu'il implique. C'est la raison pour laquelle la légitimité de la scripte est souvent fragile. La discrétion est alors une condition nécessaire de l'accomplissement de son travail: en masquant l'empiétement ou la remise en cause de l'autonomie de l'activité créatrice que cette fonction d'interface requiert, elle en favorise aussi l'acceptabilité sociale.

\section{Déclaration d'intérêts}

L'auteur déclare ne pas avoir de conflit d'intérêts en relation avec cet article.

\section{Remerciements}

L'auteur remercie Anni Borzeix ainsi que ses collègues David Lepoutre, Albert Piette et François Vatin pour leurs lectures et suggestions. Elle remercie aussi Adeline Lamberbourg avec qui une première réflexion commune sur le métier de scripte avait été engagée (Lamberbourg et Rot, 2013).

\section{Références}

Arasse, D., 1996. Le détail. Pour une histoire rapprochée de la peinture. Flammarion, Paris.

Baker, W., Falkner, R., 1991. Role as resource in the Hollywood film industry. American Journal of Sociology 97 (2), 279-309.

Barthes, R., 1980. La chambre claire. Note sur la photographie. Cahiers du cinéma, Gallimard, Seuil, Paris.

Baudrot, S., Salvini, I., 1995. La script-girl. Cinéma/vidéo. Fémis, Paris.

Bechky, B.A., 2006. Gaffers, gofers, and grips : role-based coordination in temporary organization. Organization Science 17 (1), 3-21.

Becker, H.S., 1988. Les mondes de l'art. Flammarion, Paris.

Benghozi, P.-J., 1989. Le cinéma, entre l'art et l'argent. L'Harmattan, Paris.

Berliner, T., Cohen, D.J., 2011. The illusion of continuity : active perception and the classical editing system. Journal of Film and Video 63 (1), 44-63.

Bidet, A., Borzeix, A., Pillon, T., Rot, G., Vatin, F. (Eds.), 2006. Sociologie du travail et activité. Octarès, Toulouse.

Buchanan, A., 1951. Film making, from script to screen. Faber and Faber, London.

Burch, N., 1986. Une praxis du cinéma. Gallimard, coll. «Folio/essais », Paris.

Chabrol, C., 1999. Et pourtant je tourne. Ramsay, coll. «Poche cinéma», Paris [Première édition: Robert Lafont, $1976]$.

Chateauraynaud, F., 2004. L'épreuve du tangible. Expériences de l'enquête et surgissements de la preuve. In: Karsenti, B., Quéré, L. (Eds.), La croyance et l'enquête. Aux sources du pragmatisme. Éditions de l'EHESS, coll. «Raisons pratiques », Paris, pp. 167-194.

Dagognet, F., 1973. Écriture et iconographie. Vrin, Paris.

Darré, Y., 1986. Les créateurs dans la division du travail : le cas du cinéma d'auteur. In: Moulin, R. (Ed.), Sociologie de l'art. La Documentation française, Paris, pp. 213-222.

De Fillipi, R.J., Arthur, M.B., 1998. Paradox in project-based enterprise: the case of film making. California Management Review 40, 125-139.

Denis, J., Pontille, D., 2012. Travailleurs de l'écrit, matière de l'information. Revue d'anthropologie des connaissances 6 (1), 1-20.

Diamant-Berger, H., 1977. Il était une fois le cinéma. Éditions Jean-Claude Simoën, Paris.

Dodier, N., 1995. Les hommes et les machines. La conscience collective dans les sociétés technicisées. Métailié, Paris. 
Ebbers, J., Wijnberg, N.M., 2009. Latent organizations in the film industry: contracts, rewards and resources. Human Relations 62 (7), 987-1009.

Elias, N., 1985. La société de cour. Flammarion, Paris [première édition : 1969].

Fraenkel, B., 2006. Actes écrits, actes oraux : la performativité à l'épreuve de l'écriture. Études de communication 29, 69-93.

Friedberg, E., 1993. Le pouvoir et la règle. Dynamiques de l'action organisée. Seuil, Paris,

Friedmann, G., 1950. Où va le travail humain? Gallimard, Paris.

Friedmann, G., Morin, E., 1952. Sociologie du cinéma. Revue internationale de filmologie 10, 95-112.

Gardey, D., 2010. Scriptes de la démocratie : les sténographes et rédacteurs des débats (1848-2005). Sociologie du travail $52(2), 195-211$.

Gaudy, C., 2008. Être femme sur un plateau de tournage. Ethnologie française 1, 107-117.

Glevarec, H., 2001. France culture à l'œuvre. CNRS Éditions, Paris.

Goffman, E., 1974. Les cadres de l'expérience. Éditions de Minuit, Paris.

Goodwin, C., 1994. Professional vision. American Anthropologist, New Series 96 (3), 606-633.

Grimaud, E., 2004. Bollywood film studio ou comment les films se font à Bombay. CNRS Éditions, Paris.

Hughes, E.C., 1996. Le regard sociologique. Essais sociologiques, textes rassemblés et présentés par Jean-Michel Chapoulie. Éditions de l'EHESS, Paris.

Hutchins, E., 1994. Comment le « cockpit» se souvient de ses vitesses. Sociologie du travail 36 (4), 451-473.

Joseph, I., 1994. Attention distribuée et attention focalisée. Les protocoles de la coopération au PCC de la ligne A du RER. Sociologie du travail 36 (4), 563-585.

Lahire, B., 1997. Masculin-féminin. L'écriture domestique. In: Fabre, D. (Ed.), Par écrit. Ethnologie des écritures quotidiennes. Éditions de la maison des sciences de l'homme, Paris, pp. 145-165.

Lamberbourg, A., Rot, G., 2013. La mémoire de la scripte. In: Rot, G., de Verdalle, L. (Eds.), Le cinéma. Travail et organisation. La Dispute, Paris, pp. 197-211.

Mackendrick, A., 2010. La fabrique du cinéma. L'Arche, Paris.

Menger, P.-M., 2009. Le travail créateur. Éditions de l'EHESS, Seuil, Gallimard, coll. « Hautes études », Paris.

Mintzberg, H., 1982. Structure et dynamique des organisations. Les éditions d'organisation, Paris [Première édition anglaise: 1979].

Mintzberg, H., McHugh, A., 1985. Strategy formation in an adhocracy. Administrative Science Quarterly 30 (2), $160-197$.

Nahoum-Grappe, V., 1998. L'échange des regards. Terrain 30, 67-82.

Piette, A., 1996. Ethnographie de l'action. L'observation des détails. Métailié, Paris.

Pinto, J., 1990. Une relation enchantée : la secrétaire et son patron. Actes de la recherche en sciences sociales 84, 32-48.

Rot, G., 2007. Le travail dans le cinéma. Note de recherche. Raison présente 162, 7-18.

Rot, G., de Verdalle, L., 2013. Le cinéma. Travail et organisation. La Dispute, Paris.

Seroussi, B., 2006. La création au travail. Le film, produit d'un collectif. In: Bidet, A., Borzeix, A., Pillon, T., Rot, G., Vatin, F. (Eds.), Sociologie du travail et activité. Octarès, Toulouse, pp. 85-100.

Shapin, S., 1989. The invisible technician. American Scientist 77, 554-563.

Star, S.L., Strauss, A., 1999. Layers of silence, arenas of voice: the ecology of visible and invisible work. Computer Supported Cooperative Work (CSCW) 8 (1), 9-30.

Storper, M., 1989. The transition to flexible specialization in the U.S. film industry: external economies, the division of labour, and the crossing of industrial divides. Cambridge Journal of Economics 13 (2), 273-303.

Thévenot, L., 2006. L'action au pluriel. Sociologie des régimes d'engagement. La Découverte, Paris.

Uzzi, B., Spiro, J., 2005. Collaboration and creativity: the small world problem. The American Journal of Sociology 111 (2), 447-504.

Vatin, F. (Ed.), 2013. Évaluer et valoriser. Une sociologie économique de la mesure. Presses universitaires du Mirail, Toulouse [nouvelle édition revue et augmentée; date de la première édition : 2009].

Weldon, M., 1946. The script supervisor. Hollywood Quaterly 1 (3), 331-333.

Witta, J., 1949. La script-girl. In: Marion, D. (Ed.), Le cinéma par ceux qui le font. Librairie Arthème Fayard, Paris, pp. $146-161$. 